\title{
Excitonic Giant Zeeman Effect in Wide Gap Diluted Magnetic Semiconductors Based on $\mathrm{ZnO}$ and $\mathrm{GaN}$
}

\author{
W. Pacuski ${ }^{a, b}$, D. Ferrand ${ }^{b}, \mathrm{P} . \mathrm{Kossacki}^{a, b}, \mathrm{~S} \mathrm{MARCET}^{b}$, \\ J. CiberT ${ }^{c}$, J.A. GAJ ${ }^{a}$ AND A. GolniK ${ }^{a}$ \\ ${ }^{a}$ Institute of Experimental Physics, Warsaw University \\ Hoża 69, 00-681, Warszawa, Poland \\ ${ }^{b}$ CNRS-CEA-UJF group "Nanophysique et Semiconducteurs" \\ BP 87, 38402, St Martin d'Hères Cedex, France \\ ${ }^{c}$ Laboratoire Louis Néel, CNRS \\ BP 166, 38042 Grenoble Cedex 9, Grenoble, France
}

\begin{abstract}
We present a theoretical description of the excitonic giant Zeeman effect observed in wide gap diluted magnetic semiconductors ( $\mathrm{Zn}, \mathrm{Co}) \mathrm{O}$ and (Ga,Mn)N. In these materials, $A$ and $B$ excitons present quite complex energy shifts and change of oscillator strengths under magnetic field. These features can be well reproduced using an excitonic Hamiltonian, taking into account ion-carrier exchange, wurtzite trigonal crystal field, spin-orbit and electron-hole exchange interactions.
\end{abstract}

PACS numbers: 75.50.Pp, 75.30.Hx, 78.20.Ls, 71.35.Ji

\section{Introduction}

$\mathrm{ZnO}$ and $\mathrm{GaN}$ are very promising wide gap semiconductors with a great potential for applications in photonics. It was predicted theoretically that magnetic ions introduced into $\mathrm{ZnO}$ or GaN interact strongly with band carriers due to $s, p-d$ interactions $[1,2]$. This is the reason, why $\mathrm{ZnO}$ and GaN based diluted magnetic semiconductors (DMSs) were expected to have high Curie temperatures and were considered as good candidates for applications in spintronics [1]. These predictions were supported by the first reports of room temperature ferromagnetism observed through magnetometry, as well as by the enhanced magneto-optical effects observed in magnetic circular dichroism spectra [3].

Despite the effort stimulated by expectations and early reports, a quantitative description of magnetic and magneto-optical properties of $\mathrm{ZnO}$ and $\mathrm{GaN}$ 
based DMSs has not been easy to achieve. Host materials, $\mathrm{ZnO}$ and GaN, are semiconductors with a wurtzite structure, a weak spin-orbit coupling and strong excitonic electron-hole exchange interactions. This combination of properties is at the origin of some controversies in the determination of band edge parameters such as excitonic $g$-factors or even of the symmetry of valence band edges in the case of $\mathrm{ZnO}$. The presence of magnetic ions such as cobalt and manganese leads to a giant Zeeman effect, which is not straightforward to describe. $A, B$, and $C$ excitons anti-cross and their transition energies and oscillator strengths are strongly affected by the magnetic field.

This paper presents problems and constructive solutions related to the study of giant Zeeman effect in wide band gap DMSs of this kind.

\section{Calculation of the giant Zeeman effect}

We follow the description of band edges given by Julier et al. for GaN [4]. We note $H_{\mathrm{V}}$ the $6 \times 6$ effective Hamiltonian describing the top of the valence band and use its conventional parametrization by $\Delta_{1}$ the trigonal crystal field parameter and $\Delta_{2}\left(\Delta_{3}\right)$ the spin-orbit parameter parallel (perpendicular) to the wurtzite $c$-axis.

After Julier et al. [4] and using the Bloch functions for valence electron, $|X \uparrow\rangle,|\mathrm{i} Y \uparrow\rangle,|Z \downarrow\rangle,|X \downarrow\rangle|-\mathrm{i} Y \downarrow\rangle,|-Z \uparrow\rangle, H_{\mathrm{V}}$ can be written in the following form:

$$
H_{\mathrm{V}}^{(0)}=\left(\begin{array}{cc}
H_{3 \mathrm{~V}} & 0 \\
0 & H_{3 \mathrm{~V}}
\end{array}\right)
$$

where

$$
H_{3 \mathrm{~V}}=\left(\begin{array}{ccc}
-\Delta_{2} & \Delta_{2} & \Delta_{3} \\
\Delta_{2} & -\Delta_{2} & -\Delta_{3} \\
\Delta_{3} & -\Delta_{3} & -\Delta_{1}-\Delta_{2}
\end{array}\right) .
$$

For magnetic field applied parallel to the $c$-axis, the spin carrier exchange interactions induced by the magnetic ions can be easily incorporated using a mean field $6 \times 6$ diagonal Hamiltonian $H_{\mathrm{GV}}$, parametrized by the hole-spin carrier exchange integral $N_{0} \beta$, the concentration of magnetic ions $x$ and the mean spin $\left\langle S_{z}\right\rangle$ carried by each magnetic ion [5]:

$$
\begin{aligned}
& H_{\mathrm{V}}=H_{\mathrm{V}}^{(0)}+H_{\mathrm{GV}}= \\
& H_{\mathrm{V}}^{(0)}+\frac{1}{2} N_{0} \beta x\left\langle-S_{z}\right\rangle\left(\begin{array}{cccccc}
1 & 0 & 0 & 0 & 0 & 0 \\
0 & 1 & 0 & 0 & 0 & 0 \\
0 & 0 & -1 & 0 & 0 & 0 \\
0 & 0 & 0 & -1 & 0 & 0 \\
0 & 0 & 0 & 0 & -1 & 0 \\
0 & 0 & 0 & 0 & 0 & 1
\end{array}\right) .
\end{aligned}
$$

Using a Bloch function basis $|S \uparrow\rangle,|S \downarrow\rangle$ for the conduction band, a $2 \times 2$ mean field Hamiltonian $H_{\mathrm{GC}}$, parametrized by the electron spin carrier exchange 
integrals $N_{0} \alpha$, can be similarly defined for the conduction band [5]

$$
H_{\mathrm{C}}=E_{0}+H_{\mathrm{GC}}=E_{0}+\frac{1}{2} N_{0} \alpha x\left\langle-S_{z}\right\rangle\left(\begin{array}{cc}
1 & 0 \\
0 & -1
\end{array}\right) .
$$

Following Julier et al. [4], a $12 \times 12$ excitonic Hamiltonian $H_{\sigma}$ can be derived by combining the 4 previous Hamiltonians and by taking into account the electronhole exchange interactions, parameterized by the exchange energy $\gamma$.

In the following, we will restrict the discussion to $\Gamma_{5}$ excitonic states optically coupled to the light in $\sigma$ polarization (light polarization perpendicular to the $c$-axis). These states are observed experimentally in Faraday configuration, when the magnetic field and the light propagation are parallel to the $c$-axis.

Using the following basis of Bloch functions associated to $\Gamma_{5}$ excitonic states, $|X \uparrow, S \uparrow\rangle,|\mathrm{i} Y \uparrow, S \uparrow\rangle,|Z \downarrow, S \uparrow\rangle,|X \downarrow, S \downarrow\rangle,|-\mathrm{i} Y \downarrow, S \uparrow\rangle,|-Z \uparrow, S \uparrow\rangle, H_{\sigma}$ can be written as follows:

$$
\begin{aligned}
& H_{\sigma}=E_{0}-H_{\mathrm{V}}^{(0)}+\frac{1}{2} N_{0} x\left\langle-S_{z}\right\rangle \\
& \times\left(\begin{array}{cccccc}
(\alpha-\beta) & 0 & 0 & 0 & 0 & 0 \\
0 & (\alpha-\beta) & 0 & 0 & 0 & 0 \\
0 & 0 & (\alpha+\beta) & 0 & 0 & 0 \\
0 & 0 & 0 & -(\alpha-\beta) & 0 & 0 \\
0 & 0 & 0 & 0 & -(\alpha-\beta) & 0 \\
0 & 0 & 0 & 0 & 0 & -(\alpha+\beta)
\end{array}\right) \\
& +\frac{\gamma}{2}\left(\begin{array}{cccccc}
-1 & 0 & 0 & 2 & 0 & 0 \\
0 & -1 & 0 & 0 & -2 & 0 \\
0 & 0 & 1 & 0 & 0 & 0 \\
2 & 0 & 0 & -1 & 0 & 0 \\
0 & -2 & 0 & 0 & -1 & 0 \\
0 & 0 & 0 & 0 & 0 & 1
\end{array}\right)
\end{aligned}
$$

The transition energy of the $\Gamma_{5}$ excitonic eigenstates $|\psi\rangle$ can be calculated by diagonalizing the Hamiltonian (5) for a given value of the mean spin $\left\langle-S_{z}\right\rangle$ of the magnetic ions. The oscillator strength in each circular polarization is given by the projection of $|\psi\rangle$ on the relevant subspace:

$$
\frac{1}{2}|\langle X \uparrow, S \uparrow \mid \psi\rangle-\langle\mathrm{i} Y \uparrow, S \uparrow \mid \psi\rangle+\langle X \downarrow, S \downarrow \mid \psi\rangle+\langle-\mathrm{i} Y \downarrow, S \downarrow \mid \psi\rangle|^{2} \quad \text { for } \sigma^{+}
$$

and

$$
\frac{1}{2}|\langle X \uparrow, S \uparrow \mid \psi\rangle+\langle\mathrm{i} Y \uparrow, S \uparrow \mid \psi\rangle+\langle X \downarrow, S \downarrow \mid \psi\rangle-\langle-\mathrm{i} Y \downarrow, S \downarrow \mid \psi\rangle|^{2} \quad \text { for } \sigma^{-} .
$$

In a chosen normalization the sum of oscillator strength of all transitions is equal to 2 in each circular polarization. In zero field, the eigenstates are separated into 3 doublets: $A, B$, and $C$. The lowest states ( $A$ and $B$ excitons) have a large oscillator strength in $\sigma$ polarization. The $C$ exciton, which is repelled to high energy by the trigonal crystal field, is coupled to light only by the mixing with $A$ and $B$ excitons induced by spin-orbit. If the crystal field parameter $\Delta_{1}$ is much 
larger than the spin-orbit terms $\left(\Delta_{2}, \Delta_{3}\right)$, the mixing term is weak and exciton $C$ cannot be easily observed experimentally.

If the electron-hole exchange interaction $(\gamma)$ is weak compared to spin-orbit interaction $\left(\Delta_{2}\right)$, states with parallel spins are formed, e.g. $(1 / \sqrt{2})(|X \uparrow, S \uparrow\rangle+|\mathrm{i} Y \uparrow, S \uparrow\rangle)$. If $\gamma$ is comparable to $\Delta_{2}$, there is an important mixing of states with opposite spins, e.g., $a(|X \uparrow, S \uparrow\rangle+|\mathrm{i} Y \uparrow, S \uparrow\rangle)+$ $b(|X \downarrow, S \downarrow\rangle+|\mathrm{i} Y \downarrow, S \downarrow\rangle)$. If we add the giant Zeeman effect, it is even possible to form a bright exciton: $\frac{1}{2}(|X \uparrow, S \uparrow\rangle+|\mathrm{i} Y \uparrow, S \uparrow\rangle)+\frac{1}{2}(|X \downarrow, S \downarrow\rangle+|\mathrm{i} Y \downarrow, S \downarrow\rangle)$ with maximum of oscillator strength in $\sigma^{-}$and a dark exciton, $\frac{1}{2}(|X \uparrow, S \uparrow\rangle+|\mathrm{i} Y \uparrow, S \uparrow\rangle)-$ $\frac{1}{2}(|X \downarrow, S \downarrow\rangle+|\mathrm{i} Y \downarrow, S \downarrow\rangle)$.

The valence band ordering is defined by the sign of the spin-orbit parameter $\Delta_{2}$. A positive sign corresponds to a conventional $\Gamma_{9}, \Gamma_{7}, \Gamma_{7}$ valence band ordering, and a negative one to an inverse $\Gamma_{7}, \Gamma_{9}, \Gamma_{7}$ ordering.

In the diluted limit, an analytical expression of the mean spin $\left\langle-S_{z}\right\rangle$ as a function of temperature and magnetic field can be established for $\mathrm{Co}^{2+}$ in $\mathrm{ZnO}$ [5] and $\mathrm{Mn}^{3+}$ in GaN [6]. The other parameters $\Delta_{1}, \Delta_{2}, \Delta_{3}, \gamma, N_{0} \alpha, N_{0} \beta$, and $x$ have to be determined from a fit of the experimental data for each particular sample. In this calculation, we kep the product $x\left\langle-S_{z}\right\rangle$ as a free parameter in order to maintain a general description valid for both materials and any magnetic ion composition.

\section{Results}

We performed reflectivity and absorption measurements on diluted $(\mathrm{Zn}, \mathrm{Co}) \mathrm{O}$ and $(\mathrm{Ga}, \mathrm{Mn}) \mathrm{N}$ epilayers. The measurements were done in Faraday configuration using magnetic field up to $11 \mathrm{~T}$ and temperatures down to $1.5 \mathrm{~K}$. Large shifts of $A$ and $B$ excitonic lines can be observed in both circular polarizations under magnetic field. The Zeeman splitting of the excitonic lines decreases with increasing temperature and increases nonlinearly with the magnetic field. The details of these experiments and of the determination of fitting parameters can be found in Ref. [5] for $\mathrm{ZnO}: \mathrm{Co}^{2+}$ and will be given in Ref. [7] for $\mathrm{GaN}: \mathrm{Mn}^{3+}$. For $(\mathrm{Ga}, \mathrm{Mn}) \mathrm{N}$, we use the band parameters given in Ref. [8] for pure GaN.

We found a following set of parameters $\Delta_{1}=51 \mathrm{meV}, \Delta_{2}=3 \mathrm{meV}, \Delta_{3}=$ $6.3 \mathrm{meV}, \gamma=3.4 \mathrm{meV}$, and $N_{0}(\alpha-\beta)=0.8 \mathrm{eV}$ for (Zn,Co)O and $\Delta_{1}=13 \mathrm{meV}$, $\Delta_{2}=7 \mathrm{meV}, \Delta_{3}=5.5 \mathrm{meV}, \gamma=0.6 \mathrm{meV}$, and $N_{0}(\alpha-\beta)=1.2 \mathrm{eV}$ for $(\mathrm{Ga}, \mathrm{Mn}) \mathrm{N}$. The values found for $\Delta_{1}, \Delta_{2}, \Delta_{3}$ can differ from the bulk values due to the effect of the strain of the epilayers. We assumed a usual value of $N_{0} \alpha=0.2 \mathrm{eV}$, The obtained transition energies and oscillator strengths of the excitonic lines are shown for both materials in Fig. 1a and b as a function of product $\left(x\left\langle-S_{z}\right\rangle\right)$.

As shown in Fig. 1, excitons $A$ and $B$ shift in opposite directions. They separate in $\sigma^{-}$polarization in the case of $(\mathrm{Zn}, \mathrm{Co}) \mathrm{O}$ and in $\sigma^{+}$polarization in the case of $(\mathrm{Ga}, \mathrm{Mn}) \mathrm{N}$, whereas in the opposite polarizations they approach each other. For higher magnetization, exciton $B$ interacts with exciton $C$ due to the 

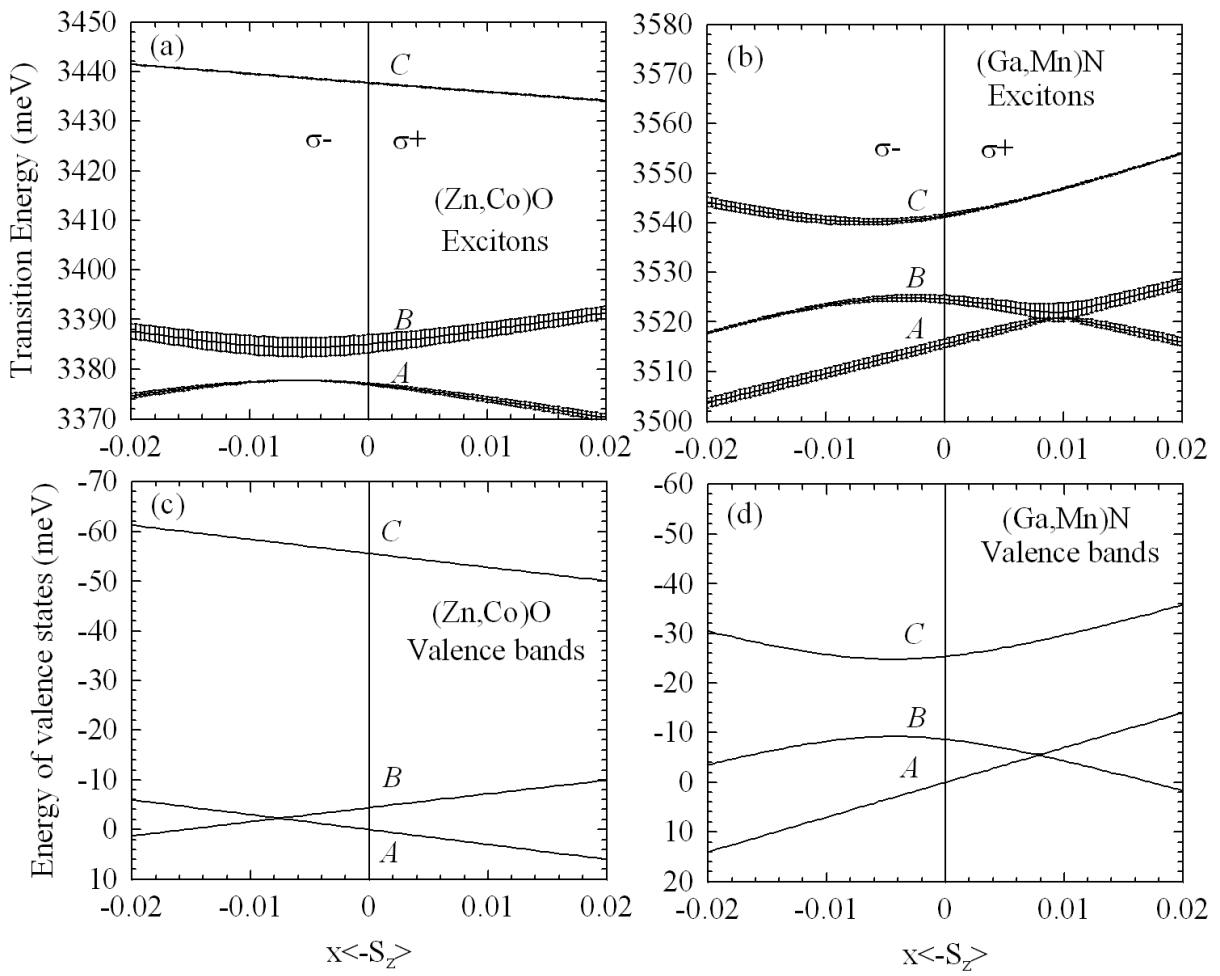

Fig. 1. Results of calculation: (a) and (b) energies of the $A, B$, and $C$ excitonic transitions versus magnetization $x\left\langle-S_{z}\right\rangle$, in $\sigma^{-}$(left half) and $\sigma^{+}$(right half) circular polarization. Bars show oscillator strength; (c) and (d) energies of the $A, B$, and $C$ valence bands edges shown in the same way as excitonic transitions. Let us note that $A$ and $B$ valence bands cross, while an anti-crossing occurs for $A$ and $B$ excitons.

spin-orbit coupling term $\Delta_{3}$. In ZnO, the separation energy $\left(\Delta_{1}-\frac{1}{2} \Delta_{2}\right)$ between excitons $B$ and $C$ is large and the experimental observation of the mixing between $B$ and $C$ excitons is difficult.

In the case of $(\mathrm{Zn}, \mathrm{Co}) \mathrm{O}$, excitons anti-cross in $\sigma^{-}$circular polarization due to the electron-hole exchange interaction. This anti-crossing significantly decreases the giant Zeeman shift of excitons $A$ and $B$. The same coupling in $(\mathrm{Ga}, \mathrm{Mn}) \mathrm{N}$ is observed in $\sigma^{+}$circular polarization and is weaker. We should note that the corresponding $A$ and $B$ hole subbands cross freely (see Figs. 1c and d). In the case of $(\mathrm{Zn}, \mathrm{Co}) \mathrm{O}$, the Zeeman shift of excitons $A$ and $B$ was found to be twice smaller than the giant Zeeman shift of bands, $x\left\langle-S_{z}\right\rangle N_{0}(\alpha-\beta)$.

The bars on the curves in Fig. 1a and b are proportional to the oscillator strengths. For $(\mathrm{Ga}, \mathrm{Mn}) \mathrm{N}$, the oscillator strengths of excitons $A$ and $B$ are almost equal in zero field. Exciton $C$ is much weaker. In $\sigma^{-}$circular polarization, the coupling between excitons $B$ and $C$ increases and we observe a transfer of oscillator 
strength from exciton $B$ to exciton $C$. Close to the anti-crossing between excitons $A$ and $B$, the upper excitonic line forms a bright exciton (with a large oscillator strength) and the lower one forms a dark exciton (with a small oscillator strength). Let us note that in all cases the total oscillator strength of $A, B$, and $C$ remains constant.

Electron-hole exchange interaction is crucial in analyzing the results obtained for $(\mathrm{Zn}, \mathrm{Co}) \mathrm{O}$. The oscillator strength is strongly affected by this interaction even in zero field. The observation of exciton $C$ is rather difficult because it has a very small oscillator strength.

In Fig. 1a we assume a usual valence band ordering $\left(\Gamma_{9}, \Gamma_{7}, \Gamma_{7} ; \Delta_{2}>0\right)$ for $\mathrm{ZnO}$, but a similar plot can be obtained using an unusual valence band ordering $\left(\Gamma_{7}, \Gamma_{9}, \Gamma_{7}\right)$ and by changing the sign of $N_{0}(\alpha-\beta)$ (not shown). The $C$ exciton is significantly affected by the sign of exchange integrals but its weak oscillator strength does not allow us to distinguish experimentally between the two assumptions.

\section{Conclusions}

We developed a model describing the excitonic giant Zeeman effect in wide band gap diluted magnetic semiconductors. The model takes into account trigonal crystal field, spin-orbit and electron-hole exchange interactions. By fitting experimental data obtained with $(\mathrm{Ga}, \mathrm{Mn}) \mathrm{N}$ and $(\mathrm{Zn}, \mathrm{Co}) \mathrm{O}$ epilayers, we estimate the difference of the spin carrier exchange integrals $N_{0}(\alpha-\beta)$. In these wide gap materials with a small spin-orbit, the giant Zeeman shifts of excitons $A$ and $B$ behave differently than those known from classical diluted magnetic semiconductors, such as $(\mathrm{Cd}, \mathrm{Mn}) \mathrm{Te}$. They depend strongly on the mixing terms induced by the spin-orbit and by electron-hole exchange interactions.

\section{Acknowledgments}

We would like to thank prof. R. Stȩpniewski and W. Bardyszewski for helpful discussions. This work was partially supported by the Ministry of Science and Higher Education (under grant 2PO3B 002 25), program Polonium and a grant from the French Ministry of Foreign Affairs.

\section{References}

[1] T. Dietl, H. Ohno, F. Matsukura, J. Cibert, D. Ferrand, Science 287, 1019 (2000).

[2] J. Blinowski, P. Kacman, T. Dietl, in: Spintronics, MRS Symp. Proc. No. 690, paper No. F6.9, Eds. T.J. Klemmer, J.Z. Sun, A. Fert, Materials Research Society, Warrendale (PA) 2001.

[3] K. Ando, J. Appl. Phys. 89, 7284 (2001); Appl. Phys. Lett. 82, 100 (2003).

[4] M. Julier, J. Campo, B. Gil, J.P. Lascaray, S. Nakamura, Phys. Rev. B 57, R6791 (1998). 
[5] W. Pacuski, D. Ferrand, J. Cibert, C. Deparis, J.A. Gaj, P. Kossacki, C. Morhain, Phys. Rev. B 73, 035214 (2006).

[6] S. Marcet, D. Ferrand, D. Halley, S. Kuroda, H. Mariette, E. Gheeraert, F.J. Teran, M.L. Sadowski, R.M. Galera, J. Cibert, cond-mat/0604025 (2006).

[7] W. Pacuski, D. Ferrand, J. Cibert, J.A. Gaj, A. Golnik, P. Kossacki, S. Marcet, H. Mariette, E. Sarigiannidou, to be published.

[8] B. Gil, O. Briot, R.L. Aulombard, Phys. Rev. B 52, R17028 (1995). 